quant-ph/9809047

\title{
Quantum mechanics of an electron in a homogeneous magnetic field and a singular magnetic flux tube
}

\author{
Hans-Peter Thienel \\ Universität Siegen, Fachbereich Physik, D-57068 Siegen, Germany \\ e-mail: thienel@hepth2.physik.uni-siegen.de
}

\begin{abstract}
The eigenvalue problem of the Hamiltonian of an electron confined to a plane and subjected to a perpendicular time-independent magnetic field which is the sum of a homogeneous field and an additional field contributed by a singular flux tube, i. e. of zero width, is investigated. Since both a direct approach based on distribution-valued operators and a limit process starting from a non-singular flux tube, i. e. of finite size, fail, an alternative method is applied leading to consistent results. An essential feature is quantum mechanical supersymmetry at $g=2$ which imposes, by proper representation, the correct choice of "boundary conditions". The corresponding representation of the Hilbert space in coordinate space differs from the usual space of square-integrable 2-spinors, entailing other unusual properties. The analysis is extended to $g \neq 2$ so that supersymmetry is explicitly broken. Finally, the singular Aharonov-Bohm system with the same amount of singular flux is analysed by making use of the fact that the Hilbert space must be the same.
\end{abstract}




\section{Introduction}

The parameter space of the system that is considered interpolates between two very different systems of fundamental importance.

On the one hand, the motion of an electron in a plane perpendicular to a homogeneous magnetic field [1] [2] has found an important application in quantum Hall physics [3] [4] [5]. Its most striking feature is the vast degeneracy within the discrete Landau levels that makes quantum Hall phenomena possible. It is of interest how this system is altered by inhomogeneities of the magnetic field. An additional singular flux tube appears to be a minimal modification of the homogeneous field [6], while in general inhomogeneous magnetic fields are notoriously difficult to handle.

On the other hand, since the work of Aharonov and Bohm [7] the physics of a magnetic flux tube alone has been investigated under a variety of aspects. Considering particles of spin $1 / 2$ [8], the system is of interest as a simple application of mathematical index theorems [9] [10] [11], interrelated with anomalies in quantum field theories [12] 13] 14 and the fact that the Pauli equation exhibits a quantum mechanical supersymmetry for $g=2$ [15]. Furthermore, it is particles of spin $1 / 2$ that problems occur for, when we go over from the non-singular, i. e. flux tube of finite width, to the singular case, i. e. flux tube of zero width. While for the nonsingular case the standard quantum mechanics works well, the singular case raises the question of the correct boundary conditions at the location of the flux tube. Standard boundary conditions, e. g. of the usual Dirichlet type, are not sufficient to characterize the behavior of the eigenfunctions of the Hamiltonian [16] [17]. This is due to the fact that functions of different boundary behavior are mapped into each other by the supersymmetry, which is thus not represented automatically by respecting simple boundary conditions. This difficulty makes one suspect that the transition from the non-singular to the singular flux tube might not be continuous. This point among other questions is obscured by the fact that a flux tube alone, yielding the free particle behavior as we go to infinity, leads to a continuum in the spectrum of the Hamiltonian entailing non-normalizable eigenfunctions.

We supply the singular flux tube of flux $\alpha \Phi$, where $\alpha$ is real and $\Phi=h c /|e|$, with an additional homogeneous magnetic field and solve the eigenvalue problem of the Hamiltonian. Technically our procedure deviates from standard methods considerably. Some features that we are familiar with from other quantum mechanical systems are not found, while other features that are unusual in a quantum mechanical context do occur. Therefore, we want to clarify some issues relevant to our system and point out some peculiarities of the system in advance.

The integral part of $\alpha$ is not a gauge effect. The similarity to a gauge transformation merely shows that an inhomogeneity constrained to a single point leaves many features of the system intact. Yet, systems differing by integral quanta of singular flux are different.

We solve the non-relativistic Pauli equation and not the corresponding Dirac equation. A relativistic treatment would introduce the Compton wave length $\lambda_{C}$

as an additional scale to the system. An infinitely thin flux tube would run into 
trouble with the consistency of one-particle quantum mechanics by introducing a scale falling below $\lambda_{C}$. While a magnetic field does not accelerate a charged particle, a gradient exerts a force on the magnetic dipole. We could be confident about the one-particle description if the radius of the flux tube were at least of the magnitude $|\alpha|^{1 / 2} \lambda_{C}$. Otherwise, pair production due to the inhomogeneity of the magnetic field had to be taken into account. Staying non-relativistic we avoid any conflicts. Clearly, this means that a singular flux tube idealizes a real flux tube of minimal radius $|\alpha|^{1 / 2} \lambda_{C}$.

The quantum system will not be realized in coordinate space before the limit $R \rightarrow 0$ is taken, where $R$ denotes the radius of the tube to which the flux $\alpha \Phi$ is confined. In the regularized coordinate representation we quantize the system and calculate matrix elements. The regularization is an intrinsic necessity in order to handle unavoidable products of singular objects at the flux tube consistently, since matrix elements contain products of both singular operators and singular wave functions. Supersymmetric pairing turns out to be possible for eigenfunctions only due to an arbitrariness following from the regularization. Also, this regularization makes possible a deviation of the Hilbert space from the space of square-integrable 2-spinors $L_{2} \otimes \mathbb{C}^{2}$ for $|\alpha| \geq 1$. In particular, singular supersinglet states occur with properties similar to those of a classical point particle in two dimensions.

Usually, after solving an eigenvalue problem of a Hamiltonian we verify that the set of eigenfunctions supplies a resolution of unity in the space of square-integrable functions [18]. For our singular system, however, we do not know a priori what the Hilbert space should be, i. e. what space of functions we should find a resolution of unity for. Conversely, we use the orthonormal set of eigenfunctions of the Hamiltonian to span the Hilbert space. The completeness of the eigenfunctions of the Hamiltonian within the Hilbert space is an indispensable requirement that is accounted for in this way by the very construction.

As opposed to non-singular systems, in the presence of a singular flux tube the representation of supersymmetry is not automatic by a standard treatment using local boundary conditions 13 . Instead, insisting on a preservation of supersymmetry uniquely determines the correct eigenfunctions of the Hamiltonian and, hence, the correct Hilbert space.

We specifically treat the electron with charge $-|e|$. The considerations apply to any elementary, charged, massive particle of spin $1 / 2$ by obvious modifications.

\section{Supersymmetry}

The following statements [19] 20] [15] rely on the assumption that all quantities are sufficiently differentiable and non-singular so that their products are defined.

We consider the Pauli Hamiltonian in the $x$-y-plane with $p_{z}=0, g=2$, and a perpendicular magnetic field $\vec{B}=B_{z}(\vec{r}) \vec{e}_{z}$, where $\vec{r}=(x, y)$

$$
H=\frac{M}{2}\left(v_{x}^{2}+v_{y}^{2}\right)+\frac{|e| \hbar}{M c} B_{z} S_{z},
$$


with the velocities $v_{x}=\left(p_{x}+\frac{|e|}{c} A_{x}\right) / M$ and $v_{y}=\left(p_{y}+\frac{|e|}{c} A_{y}\right) / M$. Angular momenta are always given in units of $\hbar$. We define the superoperators $Q=S_{+} V$ and $Q^{\dagger}=$ $S_{-} V^{\dagger}$ with $V=(M / 2)^{1 / 2}\left(v_{y}+i v_{x}\right), V^{\dagger}=(M / 2)^{1 / 2}\left(v_{y}-i v_{x}\right)$ and $S_{ \pm}:=S_{x} \pm i S_{y}$.

The Hamiltonian and the superoperators obey the supersymmetry algebra

$$
\begin{gathered}
Q^{2}=\left(Q^{\dagger}\right)^{2}=0, \quad H=Q Q^{\dagger}+Q^{\dagger} Q . \\
\Rightarrow[H, Q]=\left[H, Q^{\dagger}\right]=0 .
\end{gathered}
$$

In addition we have

$$
\left[S_{z}, Q\right]=Q, \quad\left[S_{z}, Q^{\dagger}\right]=-Q^{\dagger}, \quad\left[S_{z}, H\right]=0 .
$$

Hermitian superoperators are $Q^{(1)}=Q+Q^{\dagger}=(2 M)^{1 / 2}\left(S_{x} v_{y}-S_{y} v_{x}\right)$ and $Q^{(2)}=$ $i\left(Q^{\dagger}-Q\right)=(2 M)^{1 / 2}\left(S_{x} v_{x}+S_{y} v_{y}\right)$.

We can immediately draw important conclusions from supersymmetry. On the one hand, the measured energy is

$$
\langle\Psi|H| \Psi\rangle=\langle Q \Psi \mid Q \Psi\rangle+\left\langle Q^{\dagger} \Psi \mid Q^{\dagger} \Psi\right\rangle \geq 0 .
$$

On the other hand, any eigenstate of the Hamiltonian with eigenvalue $E>0$ is doubly degenerate. By (3) we can always arrange the partner states to be eigenstates of $S_{z}$ such that

$$
\begin{aligned}
Q \mid E, \sigma & =-1 / 2\rangle \sim|E, \sigma=+1 / 2\rangle, \\
Q^{\dagger} \mid E, \sigma & =+1 / 2\rangle \sim|E, \sigma=-1 / 2\rangle,
\end{aligned}
$$

where $S_{z}|\sigma\rangle=\sigma|\sigma\rangle$ with $\sigma= \pm 1 / 2$, if $E>0$. The eigenstates of $H$ with $E=0$, being annihilated by the superoperators

$$
Q|E=0\rangle=Q^{\dagger}|E=0\rangle=0,
$$

are supersinglets.

\section{Insufficient approaches to the problem}

We give a brief sketch of two obvious approaches in order to work out their shortcomings resolved by the correct method in the next section.

\subsection{The direct approach}

We consider the magnetic field in the form $\vec{B}(\vec{r})=\left(B+\alpha \Phi \delta^{2}(\vec{r})\right) \vec{e}_{z}[6] . \quad B>0$ is constant everywhere. The corresponding vector potential is $\vec{A}(\vec{r})=(B r / 2+$ $\alpha \Phi / 2 \pi r) \vec{e}_{\varphi}$, where $r$ and $\varphi$ denote polar coordinates in the $x$-y-plane; i. e. the flux tube part is a pure gauge for $r \neq 0$ locally. We measure lengths in terms of $\lambda=(\Phi / \pi B)^{1 / 2}$ putting $\tilde{r}=r / \lambda$. Energy is measured in units of $\hbar \omega$ with the Larmor frequency $\omega=|e| B / M c$ of the homogeneous field. The superoperators are measured 
in units of $(\hbar \omega)^{1 / 2}$. For $\alpha=0$ we obtain the operators for the homogeneous magnetic field.

The Hamiltonian is

$$
H_{\alpha}=-\frac{1}{4}\left(\frac{1}{\tilde{r}} \frac{\partial}{\partial \tilde{r}} \tilde{r} \frac{\partial}{\partial \tilde{r}}+\frac{1}{\tilde{r}^{2}} \frac{\partial^{2}}{\partial \varphi^{2}}\right)+\frac{1}{4} \tilde{r}^{2}-\frac{i}{2} \frac{\partial}{\partial \varphi}+S_{z}+\frac{\alpha}{2}-\frac{i \alpha}{2 \tilde{r}^{2}} \frac{\partial}{\partial \varphi}+\frac{\alpha^{2}}{4 \tilde{r}^{2}}+\frac{\alpha}{2 \tilde{r}} \delta(\tilde{r}) S_{z}
$$

Since $\left[H_{\alpha}, L_{z}\right]=\left[H_{\alpha}, S_{z}\right]=0$, we put $\Psi_{E, \sigma, m}(\tilde{r}, \varphi)=\psi_{E, \sigma, m}(\tilde{r}) e^{i m \varphi} \zeta_{\sigma}$ in the eigenvalue equation, where $m$ is integer and $\zeta_{\sigma}$ are unit eigenspinors of $S_{z}$. For $\tilde{r} \neq 0$ the eigenvalue equation is the same as for a homogeneous magnetic field by the substitution $m \rightarrow m+\alpha$. From the eigenfunctions of the homogeneous field $\alpha=0$ [1], we know the solutions for $\alpha \neq 0$ in the entire plane except at $\tilde{r}=0$. The delta function allows only eigenfunctions to be continued to $\tilde{r}=0$ that vanish there. Accordingly, the solutions are

$$
\begin{aligned}
& \Psi_{E, \sigma, m}(\tilde{r}, \varphi)=\frac{1}{\lambda \sqrt{\pi}} \sqrt{\frac{(E-\sigma-1 / 2-(m+\alpha) \theta(m+\alpha)) !}{\Gamma(E-\sigma+1 / 2-(m+\alpha) \theta(-m-\alpha))}} \\
& \quad \times \tilde{r}^{|m+\alpha|} L_{E-\sigma-1 / 2-(m+\alpha) \theta(m+\alpha)}^{|m+\alpha|}\left(\tilde{r}^{2}\right) e^{\frac{-\tilde{r}^{2}}{2}} e^{i m \varphi} \zeta_{\sigma}
\end{aligned}
$$

with $E-\sigma-1 / 2-(m+\alpha) \theta(m+\alpha)=0,1,2, \ldots$ and $|m+\alpha| \neq 0$. For consistency with supersymmetry it is necessary that each eigenfunction with $E>0$ find a superpartner. The superoperators

$$
Q_{\alpha}=S_{+} V_{\alpha}=S_{+} \frac{e^{-i \varphi}}{2}\left(\frac{\partial}{\partial \tilde{r}}-\frac{i}{\tilde{r}} \frac{\partial}{\partial \varphi}+\tilde{r}+\frac{\alpha}{\tilde{r}}\right)
$$

and

$$
Q_{\alpha}^{\dagger}=S_{-} V_{\alpha}^{\dagger}=S_{-} \frac{e^{i \varphi}}{2}\left(-\frac{\partial}{\partial \tilde{r}}-\frac{i}{\tilde{r}} \frac{\partial}{\partial \varphi}+\tilde{r}+\frac{\alpha}{\tilde{r}}\right)
$$

however, raise or lower the leading power for small $\tilde{r}$ by one unit such that an eigenfunction (8) that is $O\left(\tilde{r}^{u}\right)$ with $0<u \leq 1$ might be mapped to a superpartner that is $O\left(\tilde{r}^{u-1}\right)$, which is not an admissible eigenfunction of $H_{\alpha}$, being in conflict with the delta function. Insisting on supersymmetry, we have to exclude all eigenfunctions in (8) of which the superpartner is not contained among the eigenfunctions in (8) as well.

For each spin component for non-integer $\alpha$ one value of $(m+\sigma)$ is missing. For integer $\alpha \neq 0$ even two values of $(m+\sigma)$ are missing. The set of eigenfunctions of the Hamiltonian certainly does not provide a complete basis for the space of squareintegrable 2-spinors $L_{2} \otimes \mathbb{C}^{2}$ if $\alpha \neq 0$, because of the missing $m$-values. Although it appears strange at first sight, this is no reason to reject this approach. One should also recall that the canonical orbital angular momentum $L_{z}$ is not a gauge-covariant operator and, therefore, is not observable. The eigenstates of this direct approach are contained among the correct eigenstates displayed in figure 1. However, for $\alpha>0$ the column of states left to the defect line and for $\alpha<0$ the column right to 
the defect line is missing. Also the singular states given in the figure are not visible by the direct approach. Incidentally, if there were no pairing, this direct approach would still yield a vacancy for $\alpha= \pm 1, \pm 2, \ldots$.

It is not obvious that $\left\{Q_{\alpha}, Q_{\alpha}^{\dagger}\right\}=H_{\alpha}$, i. e. that the Hamiltonian with the delta function as in (7) is indeed a result of the anti-commutator. This result can be obtained using complex coordinates $Z=\tilde{r} e^{i \varphi}$, defined also for $\tilde{r}=0$ by using the identity

$$
\frac{\partial}{\partial Z} \frac{1}{Z^{*}}=\lambda^{2} \pi \delta^{2}(\vec{r})
$$

valid on functions that are continuous at $\tilde{r}=0$ [21]. The identity is derived by using a regularization that cannot be avoided. In general, products of singular operators such as $Q_{\alpha}, Q_{\alpha}^{\dagger}$, and $H_{\alpha}$ require a regularization.

Distribution-valued operators require sufficiently well-behaved wave functions playing the role of test-functions so that matrix elements are always defined. This is, implicitly, the idea behind the above approach to the eigenvalue problem of $H_{\alpha}$, where the possibility that the wave functions themselves could exhibit singular behavior is excluded by inconsistency with the formulation of the problem in terms of distribution-valued operators. An extension beyond this setting requires a regularization for both the operators and the wave functions in order to make the corresponding products defined. A treatment of the eigenvalue problem of $H_{\alpha}$ based on such a regularization will have to reproduce all eigenfunctions of the above method, which are certainly correct. It could still, however, reveal other more singular eigenfunctions.

\subsection{The singular flux tube as a limiting case of a flux tube of finite size}

Now we consider the system of a cylindrical flux tube of finite radius $R$. Apart from the homogeneous field throughout the plane, we add another homogeneous field for $r \leq R$ contributing the flux $\alpha \Phi$. The magnetic field is

$$
\vec{B}(\vec{r})=\left[B+\theta(R-r) \alpha \Phi / \pi R^{2}\right] \vec{e}_{z},
$$

where $\theta(R-r)=1$ for $r \leq R$ and 0 otherwise. The corresponding vector potential is

$$
\vec{A}(\vec{r})=\left[\frac{B r}{2}+\theta(r-R) \frac{\alpha \Phi}{2 \pi r}+\theta(R-r) \frac{\alpha \Phi r}{2 \pi R^{2}}\right] \vec{e}_{\varphi}
$$

We employ $\tilde{r}=r / \lambda$ and $\tilde{R}=R / \lambda$ with the magnetic length $\lambda$ of the homogeneous field outside of the flux tube and the corresponding $\hbar \omega$ as the unit of energy. The Hamiltonian is given by

$$
H_{\alpha}=-\frac{1}{4}\left(\frac{1}{\tilde{r}} \frac{\partial}{\partial \tilde{r}} \tilde{r} \frac{\partial}{\partial \tilde{r}}+\frac{1}{\tilde{r}^{2}} \frac{\partial^{2}}{\partial \varphi^{2}}\right)+\frac{1}{4} \tilde{r}^{2}-\frac{i}{2} \frac{\partial}{\partial \varphi}+S_{z}
$$




$$
+\theta(\tilde{r}-\tilde{R})\left(\frac{\alpha}{2}-\frac{i \alpha}{2 \tilde{r}^{2}} \frac{\partial}{\partial \varphi}+\frac{\alpha^{2}}{4 \tilde{r}^{2}}\right)+\theta(\tilde{R}-\tilde{r}) \frac{\alpha}{\tilde{R}^{2}}\left(\frac{\alpha}{4 \tilde{R}^{2}} \tilde{r}^{2}+\frac{1}{2} \tilde{r}^{2}-\frac{i}{2} \frac{\partial}{\partial \varphi}+S_{z}\right)
$$

and the superoperators are given by

$$
\begin{gathered}
Q_{\alpha}=S_{+} V_{\alpha}=S_{+} \frac{e^{-i \varphi}}{2}\left[\frac{\partial}{\partial \tilde{r}}-\frac{i}{\tilde{r}} \frac{\partial}{\partial \varphi}+\tilde{r}+\frac{\alpha}{\tilde{r}}+\alpha \theta(\tilde{R}-\tilde{r})\left(\frac{\tilde{r}}{\tilde{R}^{2}}-\frac{1}{\tilde{r}}\right)\right], \\
Q_{\alpha}^{\dagger}=S_{-} V_{\alpha}^{\dagger}=S_{-} \frac{e^{i \varphi}}{2}\left[-\frac{\partial}{\partial \tilde{r}}-\frac{i}{\tilde{r}} \frac{\partial}{\partial \varphi}+\tilde{r}+\frac{\alpha}{\tilde{r}}+\alpha \theta(\tilde{R}-\tilde{r})\left(\frac{\tilde{r}}{\tilde{R}^{2}}-\frac{1}{\tilde{r}}\right)\right] .
\end{gathered}
$$

The supersymmetry algebra (2) is fulfilled. By $\left[H_{\alpha}, S_{z}\right]=\left[H_{\alpha}, L_{z}\right]=0$ we put $\Psi_{E, \sigma, m}(\tilde{r}, \varphi)=\psi_{E, \sigma, m}(\tilde{r}) e^{i m \varphi} \zeta_{\sigma}$ for the eigenfunction. The eigenvalue equation of $H_{\alpha}$ supplies differential equations of Kummer type both inside and outside of the flux tube. The inside solution has to be regular at $\tilde{r}=0$, while the outside solution must decay as $\tilde{r} \rightarrow \infty$. We match the two solutions by demanding continuity at $\tilde{r}=\tilde{R}$ yielding

$$
\begin{gathered}
\Psi_{E, \sigma, m}(\tilde{r}, \varphi) \sim\left[\theta(\tilde{r}-\tilde{R}) \tilde{r}^{m+\alpha} e^{\frac{-\tilde{r}^{2}}{2}} U\left(m+\alpha+\sigma+1 / 2-E, m+\alpha+1, \tilde{r}^{2}\right)\right. \\
+\theta(\tilde{R}-\tilde{r}) \frac{\tilde{R}^{m+\alpha-|m|} U\left(m+\alpha+\sigma+1 / 2-E, m+\alpha+1, \tilde{R}^{2}\right) e^{\frac{\alpha}{2}}}{{ }_{1} F_{1}\left(m \theta(m)+\sigma+1 / 2-\frac{\tilde{R}^{2} E}{\tilde{R}^{2}+\alpha}, 1+|m|, \tilde{R}^{2}+\alpha\right)} \\
\left.\times e^{-\left(1+\alpha / \tilde{R}^{2}\right) \tilde{r}^{2} / 2} \tilde{r}^{|m|}{ }_{1} F_{1}\left(m \theta(m)+\sigma+1 / 2-\frac{\tilde{R}^{2} E}{\tilde{R}^{2}+\alpha}, 1+|m|,\left(1+\frac{\alpha}{\tilde{R}^{2}}\right) \tilde{r}^{2}\right)\right] e^{i m \varphi} \zeta_{\sigma}
\end{gathered}
$$

with the Tricomi function $U$ [22]. Still we have to demand continuity of the first derivative at $\tilde{r}=\tilde{R}$. This entails the following equation determining the eigenvalue $E$ for given values of $\sigma, m, \alpha$, and $\tilde{R}$.

$$
\begin{gathered}
\tilde{R}^{2} \frac{U^{\prime}\left(m+\alpha+\sigma+1 / 2-E, m+\alpha+1, \tilde{R}^{2}\right)}{U\left(m+\alpha+\sigma+1 / 2-E, m+\alpha+1, \tilde{R}^{2}\right)} \\
-\left(\tilde{R}^{2}+\alpha\right) \frac{{ }_{1} F_{1}{ }^{\prime}\left(m \theta(m)+\sigma+1 / 2-\frac{\tilde{R}^{2} E}{\tilde{R}^{2}+\alpha}, 1+|m|, \tilde{R}^{2}+\alpha\right)}{{ }_{1} F_{1}\left(m \theta(m)+\sigma+1 / 2-\frac{\tilde{R}^{2} E}{\tilde{R}^{2}+\alpha}, 1+|m|, \tilde{R}^{2}+\alpha\right)}+m \theta(-m)+\alpha=0 .
\end{gathered}
$$

The prime indicates differentiation with respect to the third argument.

Since we are interested in the limit $\tilde{R} \rightarrow 0$ we consider (17) for small $\tilde{R}$ and find the condition

$$
\begin{gathered}
( \pm m \pm \alpha+\alpha-|m|){ }_{1} F_{1}(m \theta(m)+\sigma+1 / 2,1+|m|, \alpha) \\
-2 \alpha_{1} F_{1}{ }^{\prime}(m \theta(m)+\sigma+1 / 2,1+|m|, \alpha)=0
\end{gathered}
$$

up to terms that vanish as $\tilde{R} \rightarrow 0$ if $\tilde{R}^{m+\alpha} U\left(m+\alpha+\sigma+1 / 2-E, m+\alpha+1, \tilde{R}^{2}\right)=$ $O\left(\tilde{R}^{ \pm(m+\alpha)}\right)$. First of all, we observe that (18) is independent of $E$. But considering 
the various cases yields a further simple result. For $\sigma=-1 / 2$ and $m \leq 0$ on the one hand, and $\sigma=+1 / 2$ and $m \geq 0$ on the other hand, (18) is identically fulfilled. Otherwise (18) is never fulfilled. Consequently, if one superpartner is in agreement with (18), the other necessarily violates (18). Thus, supersymmetric pairs are not admitted and only supersinglets can occur. Therefore, only eigenfunctions with $E=0$ are possible. They have the particularly simple form

$\Psi_{E=0, \sigma=-1 / 2, m}(\tilde{r}) \sim\left[\theta(\tilde{r}-\tilde{R}) \tilde{r}^{-(m+\alpha)} e^{\frac{-\tilde{r}^{2}}{2}}+\theta(\tilde{R}-\tilde{r}) \tilde{R}^{-\alpha} e^{\frac{\alpha}{2}} e^{-\left(1+\alpha / \tilde{R}^{2}\right) \tilde{r}^{2} / 2} \tilde{r}^{-m}\right] e^{i m \varphi} \zeta_{-1 / 2}$,

for all $\tilde{R}$ and $\alpha$ with $m=0,-1,-2, \ldots$ They can also be found by directly solving $Q_{\alpha} \Psi_{E=0}(\tilde{r}, \varphi)=Q_{\alpha}^{\dagger} \Psi_{E=0}(\tilde{r}, \varphi)=0$. These eigenfunctions are the only ones that survive the limit $\tilde{R} \rightarrow 0$. Since they can always be normalized so that their norm is finite in the limit $\tilde{R} \rightarrow 0$, all of them have to be considered as correct.

For $\alpha>0$ these singlets have quantum numbers for which no eigenfunctions are found by the direct approach. By contrast, this approach has not provided the majority of eigenfunctions found to be correct by the direct approach. Thus, there is a mutual incompatibility of the two approaches of this section. The correct method will have to reproduce the safe results of both alternatives.

We point out that the failure of the approach of a shrinking flux tube is not a result of its rough implementation. If the magnetic field profile is made smooth by a modification within the annulus between $R-\eta$ and $R$, the limit $R \rightarrow 0$ will lead to the same results.

\section{A consistent method}

\subsection{The eigenvalue problem of the Hamiltonian}

The direct approach in 3.1 suggests that a regularization should be applied. However, the most obvious way to regularize the problem, by regarding the system of the singular flux tube as the limiting system of a sequence of systems of shrinking flux tubes in 3.2, fails. Although each member of the sequence is a consistent quantum mechanical system, a limiting system does not exist in agreement with the obviously correct solutions of the direct approach. Therefore, the idea is to replace the sequence of physical systems at finite $\tilde{R}$ by a sequence of unphysical systems at finite $\tilde{R}$. Only the limiting system is physical. In other words, for finite $\tilde{R}$ the mathematical structures cannot be interpreted in a quantum mechanical sense. A consistent quantum mechanical interpretation is possible only in the limit $\tilde{R} \rightarrow 0$. This is comparable to the use of the dimensional regularization in quantum field theory, where the system may not be interpreted in physical terms before the regulator is removed.

Before establishing the formal framework, we anticipate the essential points that suffice for a solution of the eigenvalue problem. We use the operators of the finite flux tube as given in 3.2 as their regularized form. Any matrix element of an operator 
$\Omega$ is to be calculated as

$$
\langle\Xi|\Omega| \Psi\rangle=\lim _{R \rightarrow 0} \int d^{2} r \Xi^{*}(\vec{r}) \Omega \Psi(\vec{r})
$$

where the regularized forms defined below are to be employed for $\Xi(\vec{r})$ and $\Psi(\vec{r})$ as well as for $\Omega$.

We solve the eigenvalue problem of the Hamiltonian

$$
\left\langle E^{\prime}, \sigma^{\prime}, m^{\prime}\left|H_{\alpha}-E\right| E, \sigma, m\right\rangle=0 .
$$

The solutions have to be normalizable, so we impose

$$
\lim _{R \rightarrow 0} \int d^{2} r \Psi_{E, \sigma, m}^{*}(\vec{r}) \Psi_{E, \sigma, m}(\vec{r})=1 .
$$

For a solution of (21) we use the continuous ansatz (16) as a regularized form. Putting this in (21) we get a contribution only from the discontinuity of the first derivative at $\tilde{r}=\widetilde{R}$, which has to vanish for $\tilde{R} \rightarrow 0$, such that

$$
\lim _{\tilde{R} \rightarrow 0}\left[\left.\psi_{E^{\prime}, \sigma, m}(\tilde{R}) \tilde{R} \frac{\partial}{\partial \tilde{r}} \psi_{E, \sigma, m}^{\text {in }}(\tilde{r})\right|_{\tilde{r}=\tilde{R}}-\left.\psi_{E^{\prime}, \sigma, m}(\tilde{R}) \tilde{R} \frac{\partial}{\partial \tilde{r}} \psi_{E, \sigma, m}^{\text {out }}(\tilde{r})\right|_{\tilde{r}=\tilde{R}}\right]=0,
$$

ensuring hermiticity of $H_{\alpha}$ for $\tilde{R} \rightarrow 0$. This condition is, of course, fulfilled by demanding differentiability for all finite $\tilde{R}$, which is what the second approach in section 3 was based on. Allowing, however, the expression in the square brackets in (23) to be finite for any $\tilde{R}>0$ and only demanding that it vanish in the limit $\tilde{R} \rightarrow 0$ is a weaker condition on the eigenfunctions.

A detailed investigation of (23) using the ansatz (16) may be summarized as follows. There are essentially two ways for an eigenfunction to fulfill (23). The first is that the leading power in $\tilde{R}$ is great enough to make both terms within the square brackets in (23) vanish separately as $\tilde{R} \rightarrow 0$. The second is that leading non-vanishing contributions cancel each other, while higher order terms still vanish separately. This leads again to the condition (18) to be fulfilled. Since it cannot be fulfilled for both partners of a supersymmetric pair simultaneously, it can be fulfilled either for a supersinglet, i. e. an eigenfunction for $E=0$, or for a partner of an eigenfunction that fulfills (23) in the first way.

Besides normalizability and (23) we require $E \geq 0$ and supersymmetric pairing of the eigenfunctions for $E>0$.

The pairing implies that an eigenfunction for $E>0$ fulfilling (23) is admissible only if its partner also fulfills (23). But we have to take into account that the superoperators contain a derivative in $\tilde{r}$ and will in general map a continuous function with a discontinuous first derivative at $\tilde{R}$ to a function that is discontinuous at $\tilde{R}$. It is, however, always possible to restore continuity by adding a contribution that does not change the norm, i. e. a representative of the null vector. Moreover, the behavior for $\tilde{r}<\widetilde{R}$ can always be arranged to have the eigenfunction in the form (16), which we will call the basic form of the eigenfunction. The freedom of representing the null 
vector is an important feature of the regularization and will be discussed in more detail in the next section.

The normalized eigenfunctions of Laguerre type are

$$
\begin{aligned}
& \Psi_{E, \sigma, m}(\tilde{r}, \varphi)=\frac{1}{\lambda \sqrt{\pi}} \sqrt{\frac{(E-\sigma-1 / 2-m-\alpha) !}{\Gamma(E-\sigma+1 / 2)}}\left[\theta(\tilde{r}-\tilde{R}) \tilde{r}^{m+\alpha} e^{\frac{-\tilde{r}^{2}}{2}} L_{E-\sigma-1 / 2-m-\alpha}^{(m+\alpha)}\left(\tilde{r}^{2}\right)\right. \\
& +\theta(\tilde{R}-\tilde{r}) \frac{\tilde{R}^{m+\alpha-|m|} e^{\frac{a}{2}} L_{E-\sigma-1 / 2-m-\alpha}^{(m+\alpha)}\left(\tilde{R}^{2}\right)}{F_{1}\left(m \theta(m)+\sigma+1 / 2-\frac{\tilde{R}^{2} E}{\tilde{R}^{2}+\alpha}, 1+|m|, \tilde{R}^{2}+\alpha\right)} \\
& \left.\times e^{-\left(1+\alpha / \tilde{R}^{2}\right) \tilde{r}^{2} / 2} \tilde{r}^{|m|}{ }_{1} F_{1}\left(m \theta(m)+\sigma+1 / 2-\frac{\tilde{R}^{2} E}{\tilde{R}^{2}+\alpha}, 1+|m|,\left(1+\frac{\alpha}{\tilde{R}^{2}}\right) \tilde{r}^{2}\right)\right] e^{i m \varphi} \zeta_{\sigma}
\end{aligned}
$$

for $E-\sigma-1 / 2-m-\alpha=0,1,2, \ldots$ with $m+\alpha>0$ for $\sigma=-1 / 2$ and $m<0$, and $m+\alpha>-1$ for $\sigma=+1 / 2$ and $m \geq 0$.

$$
\begin{aligned}
& \Psi_{E, \sigma, m}(\tilde{r}, \varphi)=\frac{1}{\lambda \sqrt{\pi}} \sqrt{\frac{(E-\sigma-1 / 2) !}{\Gamma(E-\sigma+1 / 2-m-\alpha)}}\left[\theta(\tilde{r}-\tilde{R}) \tilde{r}^{-m-\alpha} e^{\frac{-\tilde{r}^{2}}{2}} L_{E-\sigma-1 / 2}^{(-m-\alpha)}\left(\tilde{r}^{2}\right)\right. \\
& +\theta(\tilde{R}-\tilde{r}) \frac{\tilde{R}^{-m-\alpha-|m|} e^{\frac{a}{2}} L_{E-\sigma-1 / 2}^{(-m-\alpha)}\left(\tilde{R}^{2}\right)}{F_{1}\left(m \theta(m)+\sigma+1 / 2-\frac{\tilde{R}^{2} E}{\tilde{R}^{2}+\alpha}, 1+|m|, \tilde{R}^{2}+\alpha\right)} \\
& \left.\times e^{-\left(1+\alpha / \tilde{R}^{2}\right) \tilde{r}^{2} / 2} \tilde{r}^{|m|}{ }_{1} F_{1}\left(m \theta(m)+\sigma+1 / 2-\frac{\tilde{R}^{2} E}{\tilde{R}^{2}+\alpha}, 1+|m|,\left(1+\frac{\alpha}{\tilde{R}^{2}}\right) \tilde{r}^{2}\right)\right] e^{i m \varphi} \zeta_{\sigma}
\end{aligned}
$$

for $E-\sigma-1 / 2=0,1,2, \ldots$ with $m+\alpha<0$ for $\sigma=+1 / 2$ and $m>0$, and $m+\alpha<1$ for $\sigma=-1 / 2$ and $m \leq 0$. The eigenfunctions in (24) and (25) contain all eigenfunctions of the Hamiltonian by the direct approach. But (23) still allows further eigenfunctions of Laguerre type along with their superpartners. This reduces the vacancy in the spectra by one $(m+\sigma)$-value in comparison to the direct approach.

The other eigenfunctions fulfilling (23) are supersinglets. In order to be normalized as $\tilde{R} \rightarrow 0$ they acquire an $\tilde{R}$-dependent normalization factor that lowers the leading power in $\tilde{R}$ in (23). This very fact ensures that fulfilling (23) in lowest order is sufficient for (23) to be fulfilled to all orders, since the higher powers vanish separately. We call the eigenfunctions with an $\tilde{R}$-dependent normalization factor singular eigenfunctions.

For $E=0$ and $\sigma=-1 / 2$ we find the normalized singular eigenfunctions for $m+\alpha=1$

$$
\begin{gathered}
\Psi_{E=0, \sigma=-1 / 2, m}^{s}(\tilde{r}, \varphi)=\frac{1}{\lambda \sqrt{-\pi \ln \tilde{R}^{2}}} \\
\times\left[\theta(\tilde{r}-\tilde{R}) \tilde{r}^{-1} e^{\frac{-\tilde{r}^{2}}{2}}+\theta(\tilde{R}-\tilde{r}) \tilde{R}^{-1} e^{\alpha / 2} e^{-\left(1+\frac{\alpha}{\tilde{R}^{2}}\right) \frac{\tilde{r}^{2}}{2}}\left(\frac{\tilde{r}}{\tilde{R}}\right)^{-m}\right] e^{i m \varphi} \zeta_{-1 / 2}
\end{gathered}
$$


and for $m+\alpha>1$

$$
\begin{gathered}
\Psi_{E=0, \sigma=-1 / 2, m}^{s}(\tilde{r}, \varphi)=\frac{\tilde{R}^{m+\alpha-1}}{\lambda \sqrt{\pi\left(\frac{1}{m+\alpha-1}+\alpha^{m-1} e^{\alpha}[\Gamma(-m+1)-\Gamma(-m+1, \alpha)]\right)}} \\
\times\left[\theta(\tilde{r}-\tilde{R}) \tilde{r}^{-m-\alpha} e^{\frac{-\tilde{r}^{2}}{2}}+\theta(\tilde{R}-\tilde{r}) \tilde{R}^{-m-\alpha} e^{\alpha / 2} e^{-\left(1+\frac{\alpha}{\tilde{R}^{2}}\right) \frac{\tilde{r}^{2}}{2}}\left(\frac{\tilde{r}}{\tilde{R}}\right)^{-m}\right] e^{i m \varphi} \zeta_{-1 / 2}
\end{gathered}
$$

with the incomplete gamma function [22]. We have thus recovered all the correct eigenfunctions found within the approach in 3.2 .

For $E=0$ and $\sigma=+1 / 2$ we find still another type of singular eigenfunction. For $m+\alpha=-1$ we obtain

$$
\begin{gathered}
\Psi_{E=0, \sigma=+1 / 2, m}^{s}(\tilde{r}, \varphi)=\frac{1}{\lambda \sqrt{-\pi \ln \tilde{R}^{2}}} \\
\times\left[\theta(\tilde{r}-\tilde{R}) \tilde{r}^{-1} e^{\frac{\tilde{r}^{2}}{2}} \Gamma\left(1, \tilde{r}^{2}\right)+\theta(\tilde{R}-\tilde{r}) \tilde{R}^{-1} e^{\frac{\tilde{R}^{2}}{2}} \Gamma\left(1, \tilde{R}^{2}\right) e^{-\alpha / 2} e^{\left(1+\frac{\alpha}{\tilde{R}^{2}}\right) \frac{\tilde{r}^{2}}{2}}\left(\frac{\tilde{r}}{\tilde{R}}\right)^{m}\right] e^{i m \varphi} \zeta_{+1 / 2}
\end{gathered}
$$

and for $m+\alpha<-1$

$$
\begin{aligned}
& \Psi_{E=0, \sigma=+1 / 2, m}^{s}(\tilde{r}, \varphi)= \frac{\tilde{R}^{-m-\alpha-1}}{\lambda \sqrt{\pi\left(\frac{1}{-m-\alpha-1}+e^{-\alpha}(-\alpha)^{-m-1}[\Gamma(m+1)-\Gamma(m+1,-\alpha)]\right)}} \\
& \times\left[\theta(\tilde{r}-\tilde{R}) \tilde{r}^{m+\alpha} e^{\frac{\tilde{r}^{2}}{2}} \frac{\Gamma\left(-m-\alpha, \tilde{r}^{2}\right)}{\Gamma(-m-\alpha)}\right. \\
&\left.+\theta(\tilde{R}-\tilde{r}) \tilde{R}^{m+\alpha} e^{\frac{\tilde{R}^{2}}{2}} \frac{\Gamma\left(-m-\alpha, \tilde{R}^{2}\right)}{\Gamma(-m-\alpha)} e^{-\alpha / 2} e^{\left(1+\frac{\alpha}{\tilde{R}^{2}}\right) \frac{\tilde{r}^{2}}{2}}\left(\frac{\tilde{r}}{\tilde{R}}\right)^{m}\right] e^{i m \varphi} \zeta_{+1 / 2} .
\end{aligned}
$$

They were not anticipated in section 3 by either approach.

The spectrum of $H_{\alpha}$ is displayed in figure 1.

\subsection{The Hilbert space in the regularized coordinate repre- sentation}

The scalar product in the regularized coordinate representation is given by

$$
\langle\Xi \mid \Psi\rangle=\lim _{R \rightarrow 0} \int d^{2} r \Xi^{*}(\vec{r}) \Psi(\vec{r}),
$$

exhibiting the necessary properties. An important difference between the usual coordinate representation and the regularized coordinate representation is that in the former the null vector is represented by the function that vanishes everywhere, while in the latter also functions $N(\vec{r})$ that fulfill

$$
\lim _{R \rightarrow 0} \int d^{2} r N^{*}(\vec{r}) N(\vec{r})=0
$$


also represent the null vector. We restrict the set of functions representing the null vector to those that fulfill $\lim _{R \rightarrow 0} N(\vec{r})=0$ excluding probability densities that are different from zero on curves and points in the plane. Along with the class of functions that represent the null vector, every state is represented by an equivalence class of functions by addition of representatives of the null vector. The corresponding probability density defined by $\lim _{R \rightarrow 0} \Psi^{*}(\vec{r}) \Psi(\vec{r})$ is unique by using the basic form of a wave function defined below.

The linear space of functions with the above scalar product is still not a Hilbert space, since we have to ensure completeness; i. e. every Cauchy sequence has to converge. To this end we specify the wave functions $\Psi(\vec{r}) \in \mathcal{H}_{\alpha}$. The numerable set of eigenfunctions of $H_{\alpha}$ (24)-(29) constitutes an orthogonal normalized system with respect to the scalar product

$$
\left\langle E^{\prime}, \sigma^{\prime}, m^{\prime} \mid E, \sigma, m\right\rangle=\lim _{R \rightarrow 0} \int d^{2} r \Psi_{E^{\prime}, \sigma^{\prime}, m^{\prime}}^{*}(\vec{r}) \Psi_{E, \sigma, m}(\vec{r})=\delta_{E^{\prime} E} \delta_{\sigma^{\prime} \sigma} \delta_{m^{\prime} m} .
$$

We obtain general wave functions from linear combinations of the eigenfunctions

$$
\Psi(\vec{r})=\sum_{E, \sigma, m} c_{E, \sigma, m} \Psi_{E, \sigma, m}(\vec{r}) \quad \text { with } \sum_{E, \sigma, m} c_{E, \sigma, m}^{*} c_{E, \sigma, m}<\infty .
$$

This defines the basic form of a wave function as the sum of eigenfunctions in the basic form. Wave functions will always be given in the basic form, unless otherwise indicated. The space of functions obtained in this way is a Hilbert space by construction, since we define its elements as limiting objects of the Cauchy sequence of an increasing number of members of the sum in (33).

The basic form of a wave function $\Xi_{\text {basic }}(\vec{r})$ is obtained from an arbitrary equivalent wave function $\Xi_{\text {non-basic }}(\vec{r})$ by

$$
\Xi_{\text {basic }}(\vec{r})=\sum_{E, \sigma, m} \Psi_{E, \sigma, m}(\vec{r}) \lim _{R \rightarrow 0} \int d^{2} r^{\prime} \Psi_{E, \sigma, m}^{*}\left(\vec{r}^{\prime}\right) \Xi_{\text {non-basic }}\left(\vec{r}^{\prime}\right)
$$

This relation also expresses the fact that the eigenfunctions of the Hamiltonian supply a resolution of unity. It is this requirement that enforces the construction of the Hilbert space as presented here.

For $\alpha=0$ the Hilbert space is clearly $L_{2} \otimes \mathbb{C}^{2}$. Also for non-integer $\alpha \neq 0$ the eigenfunctions of Laguerre type provide a resolution of unity for $L_{2} \otimes \mathbb{C}^{2}[23$. For $|\alpha| \geq 1$ the singular eigenfunctions are added. For integer $\alpha \neq 0$ from the functions of Laguerre type those with $(m+\sigma)$ of the vacancy are removed.

The projection of wave functions $c_{E, \sigma, m}=\langle E, \sigma, m \mid \Psi\rangle$ in the regularized coordinate representation on the elements of the complete orthogonal system yields a matrix representation where each state is uniquely represented by the vector $\left(\left\{c_{E, \sigma, m}\right\}\right)$, e. g. the null vector is given by $(0,0, \ldots)$.

The pairing of states by supersymmetry is possible in the regularized coordinate representation only by the freedom of adding a representative of the null vector. 
E. g. the supersymmetric pairing of $\Psi_{E, \sigma=-1 / 2, m}(\vec{r})$ with $\Psi_{E, \sigma=+1 / 2, m-1}(\vec{r})$ may be expressed by

$$
\begin{gathered}
\lim _{R \rightarrow 0} \int d^{2} r \Xi^{*}(\vec{r}) Q_{\alpha} \Psi_{E, \sigma=-1 / 2, m}(\vec{r})=\lim _{R \rightarrow 0} \int d^{2} r \Xi^{*}(\vec{r})\left[Q_{\alpha} \Psi_{E, \sigma=-1 / 2, m}(\vec{r})+N(\vec{r})\right] \\
=\lim _{R \rightarrow 0} \int d^{2} r \Xi^{*}(\vec{r})( \pm \sqrt{E}) \Psi_{E, \sigma=+1 / 2, m-1}(\vec{r}),
\end{gathered}
$$

with the upper sign for eigenfunctions from (24) and the lower sign for eigenfunctions from (25). The function $Q_{\alpha} \Psi_{E, \sigma=-1 / 2, m}(\vec{r})$ with a step at $r=R$ is repaired in order to obtain $\Psi_{E, \sigma=+1 / 2, m-1}(\vec{r})$ in the basic form by addition of the appropriate $N(\vec{r})$.

\subsection{Probability densities}

At this point it is useful to discuss the modulus squared $\Psi^{*}(\vec{r}) \Psi(\vec{r})$ in the limit $R \rightarrow 0$ related to measurement as a probability density for the eigenfunctions of the Hamiltonian.

Of course, for the eigenfunctions of Laguerre type the functional form of the exterior part of the modulus squared extends to all $r \neq 0$ if $R \rightarrow 0$. So only the single point $r=0$ remains to be considered.

For $|\alpha| \geq 1$ we find that the probability density is zero at $r=0$. The vast majority of the eigenfunctions of Laguerre type vanish as $r \rightarrow 0$, yielding a density that is continuous everywhere. We have a non-continuous behavior at $r=0$ if the outside density reaches a constant value or diverges as $r \rightarrow 0$, since the probability density is still zero at $r=0$. Thus, for $|\alpha| \geq 1$ the probability densities of the non-singular wavefunctions built on eigenfunctions of the Laguerre type avoid the location of the flux tube.

By contrast, for $|\alpha|<1$ the value at $r=0$ agrees with the behavior of the exterior part as $r \rightarrow 0$, such that either the probability density is continuous everywhere or the value at $r=0$ diverges as $R \rightarrow 0$ along with the exterior part diverging as $r \rightarrow 0$.

The normalization of the singular eigenfunctions implies

$$
\lim _{R \rightarrow 0} \int_{\mathcal{G}} d^{2} r \Psi_{E, \sigma, m}^{s *}(\vec{r}) \Psi_{E, \sigma, m}^{s}(\vec{r})=1
$$

for any $\mathcal{G}$ containing $r=0$. If $\mathcal{G}$ did not contain $r=0$, the integrand would converge uniformly to zero and the integral would vanish as $R \rightarrow 0$. This means that the modulus squared of a singular eigenfunction for finite $R$ is a delta convergent function [21], i. e.

$$
\lim _{R \rightarrow 0} \Psi_{E=0, \sigma, m}^{s *}(\vec{r}) \Psi_{E=0, \sigma, m}^{s}(\vec{r})=\delta^{2}(\vec{r}),
$$

where the limit $R \rightarrow 0$ is to be taken outside of an integral. Thus, the probability density of a singular eigenfunction is that of a two dimensional point particle, concentrated at the location of the flux tube.

Singular eigenfunctions occur for $|\alpha| \geq 1$ just when the probability densities of non-singular wavefunctions avoid the flux tube as mentioned above. Although 
not being formally the reason, the fact that the probability density of the singular eigenfunctions and the eigenfunctions of Laguerre type are attributed to disjoint regions in the plane conforms nicely with the mutual orthogonality. Indeed the singular eigenfunctions are orthogonal to any function $\Xi(\vec{r})$ yielding a Taylor expansion about $r=0$

$$
\lim _{R \rightarrow 0} \int d^{2} r \Psi_{E=0, \sigma, m}^{s *}(\vec{r}) \Xi(\vec{r})=0 .
$$

From this we also conclude that $\Psi_{E=0, \sigma, m}^{s}(\vec{r})$ itself neither is a delta convergent function nor does it converge to a derivative of the delta function. Since this exploits all possibilities for generalized functions concentrated at a point [21], the corresponding functional must be zero.

We define a general singular wave function $\Psi^{s}(\vec{r})$ as a linear combination of singular eigenfunctions. It also has the properties (37) and (38) if it is normalized.

\subsection{Classification of the Hilbert spaces $\mathcal{H}_{\alpha}$}

By $\alpha$ a sequence of Hilbert spaces is parametrized. We have determined each Hilbert space by solving the eigenvalue problem of the Hamiltonian exactly for all values of $\alpha$. It is, therefore, not necessary to resort to perturbation theory. Still, in view of extensions of the system and analogous situations the question arises whether we can get arbitrarily close to the exact solution of a system with $\alpha^{\prime}=\alpha+\Delta \alpha$ by perturbation theory in $\Delta \alpha$ if the system with $\alpha$ is exactly known.

A necessary condition for this is that exact solutions of the perturbed system can be expanded in terms of the exact solutions of the unperturbed system. An exact solution of the perturbed system that is orthogonal to all exact solutions of the unperturbed system can never be reached by a perturbation series.

The real numbers that $\alpha$ can assume decompose into the isolated points $\pm 1 ; \pm 2 ; \ldots$ and the complementary open intervals $(-1,1) ;(1,2),(2,3), \ldots ;(-2,-1),(-3,-2), \ldots$ This is a result of the occurrence of the singular states and the vacancy in the $(m+\sigma)$-range for $\alpha= \pm 1, \pm 2, \ldots$ These sets represent equivalence classes with respect to the relation $\mathcal{H}_{\alpha}=\mathcal{H}_{\alpha^{\prime}}$. For any $\alpha$ and $\alpha^{\prime}$ in different equivalence classes we have $\mathcal{H}_{\alpha} \neq \mathcal{H}_{\alpha^{\prime}}$ such that there is at least one state in $\mathcal{H}_{\alpha}$ or $\mathcal{H}_{\alpha^{\prime}}$ that is orthogonal to all states of the other. For any two neighboring equivalence classes it turns out that both Hilbert spaces contain at least one state that is orthogonal to all states of the other. For these states perturbation theory fails and the above equivalence classes constitute perturbatively disjoint sectors. We summarize:

i) As expected, $\Delta \alpha$ may not be arbitrarily large for a successful application of perturbation theory; in fact $|\Delta \alpha|<2$.

ii) However, very small $\Delta \alpha$ does not guarantee successful application of perturbation theory, since $\alpha$ and $\alpha+\Delta \alpha$ could still be in different equivalence classes.

iii) The open interval $(-1,1)$ is distinguished as the interval length is exceptionally 2 , while for the other equivalence classes we have interval length of 1 or $0 . \alpha=0$ 
is the only integer that is useful as a perturbative starting point for its neighboring values. The Hilbert space is that of square-integrable 2-spinors $\mathcal{H}_{\alpha}=L_{2} \otimes \mathbb{C}^{2}[23$.

The dependence of $H_{\alpha}$ and the superoperators on $\alpha$ does not anticipate the decomposition into disjoint perturbative sectors. However, by $\alpha$ we parametrize magnetic flux, which is the global topological invariant associated with a $U(1)$ principal fiber bundle characterizing the topology of the bundle [24]. Thus, the discontinuous behavior of the Hilbert spaces reflects the differing topologies, i. e. discontinuous geometries, as $\alpha$ is varied.

\subsection{An index for the singular flux tube}

Index theorems relate the spectrum of a differential operator on a manifold to a global topological invariant. The number of eigenstates of $E=0$ with $\sigma=-1 / 2$ minus those with $\sigma=+1 / 2$ yield an index, referred to as the Witten index 20 25] in supersymmetric systems. It corresponds to the index of the euclidian Dirac operator in two dimensions, which is subject to index theorems. The Atiyah-Singer index theorem [9] for manifolds without boundary and the Atiyah-Patodi-Singer [10] index theorem for manifolds with boundary apply to compact manifolds. For the $U(1)$ case in two dimensions the Atiyah-Singer index theorem reduces to the statement that the above index equals the total flux in units of the elementary flux quantum. The index theorems are not applicable to our system as the plane is not a compact manifold. Both the above index and the magnetic flux through the plane are infinite due to the presence of the homogeneous magnetic field.

More can be said in view of the fact that in the limit $R \rightarrow 0$ both the eigenfunctions of $E=0$ and the flux may be attributed to disjoint subspaces of the plane: the point $r=0$ and its complement.

For $r \neq 0$ the eigenfunctions of Laguerre type with $E=0$ and $\sigma=-1 / 2$ that are not localized close to the flux tube can be attributed to flux quanta of the homogeneous field as for $\alpha=0$ [2].

For $r=0$ we define the index $I^{s}(\alpha)$ as the number of singular eigenfunctions with $\sigma=-1 / 2$ minus the number of singular eigenfunctions with $\sigma=+1 / 2$. If $[\alpha]$ denotes the next integer below $\alpha$, it is given by

$$
I^{s}(\alpha)=[\alpha] \theta(\alpha)-[-\alpha] \theta(-\alpha)
$$

displayed in figure 2. For integer $\alpha$ we have $I^{s}(\alpha)=\alpha$, as if we had a compact manifold as in [9].

\section{$5 \quad$ Explicit breaking of supersymmetry by $g \neq 2$}

Concerning the applicability of the results obtained so far, we are facing the fact that already in vacuo $g \approx 2.0023$ by QED corrections. Even by this tiny deviation from $g=2$ supersymmetry is explicitly broken. The question is to what extent our 
results remain valid for $g \neq 2$. We discuss only the effect of QED corrections here, but for other perturbative background effects the arguments might be analogous.

The deviation of $g$ from 2 is calculated under the assumption of a homogeneous field that is sufficiently weak, i. e. $|-\vec{\mu} \cdot \vec{B}| \ll M c^{2}[26][27][28]$. For the outside region we can assume this to be true. By contrast, inside of the flux tube the magnetic field grows as $R \rightarrow 0$. Assuming that $R \rightarrow 0$ idealizes $R \approx|\alpha|^{1 / 2} \lambda_{C}$, we have $|-\vec{\mu} \cdot \vec{B}| \approx M c^{2}$ and it is not justified to put $g \neq 2$ there. The most reasonable choice is to maintain $g=2$ within the flux tube. This may also be rephrased heuristically following the correspondence principle. A strong magnetic field implies high occupation numbers of the quantum electromagnetic field. The limit of high occupation numbers corresponds to classical behavior. As a result, contributions to QED processes from the fluctuations of the quantum electromagnetic field are suppressed.

The most obvious implementation of $g=2$ inside and $g \neq 2$ outside would be to couple the entire magnetic field with $g=2$ for $r \leq R$ and with $g \neq 2$ for $r>R$. The result would, however, be inconsistent with our regularization. On the one hand, for $|\alpha|>1$ the expectation values of the Hamiltonian with the singular eigenfunctions would not reproduce the eigenvalue. On the other hand, the regularized Hamiltonian would not yield the Hamiltonian of the homogeneous field for $\alpha=0$. Therefore, we proceed differently.

We couple $B \vec{e}_{z}$ by $g=2(1+\kappa) \neq 2$ everywhere and $\left(\alpha \Phi / \pi R^{2}\right) \vec{e}_{z}$ by $g=2$ inside of the flux tube, which amounts to a term $(1+\kappa) B S_{z}+\theta(R-r)\left(\alpha \Phi / \pi R^{2}\right) S_{z}$ in the Hamiltonian. $\alpha \Phi / \pi R^{2}$ dominates $B$ in the limit $R \rightarrow 0$ and we effectively attribute to the interior $g=2$ and to the exterior $g \neq 2$ in a consistent way. The new Hamiltonian is

$$
H_{\alpha}^{\kappa}=H_{\alpha}+\kappa S_{z}=\left\{Q_{\alpha}, Q_{\alpha}^{\dagger}\right\}+\kappa S_{z}
$$

entailing the explicit breaking of supersymmetry

$$
\left[H_{\alpha}^{\kappa}, Q_{\alpha}\right]=\kappa Q_{\alpha} \quad \text { and } \quad\left[H_{\alpha}^{\kappa}, Q_{\alpha}^{\dagger}\right]=-\kappa Q_{\alpha}^{\dagger} .
$$

A lower bound for the expectation values of $H_{\alpha}^{\kappa}$ is now $\left\langle\Psi\left|H_{\alpha}^{\kappa}\right| \Psi\right\rangle \geq-\kappa / 2$ instead of zero. For the solution of the eigenvalue problem of $H_{\alpha}^{\kappa}$ we have still $\left[H_{\alpha}^{\kappa}, L_{z}\right]=\left[H_{\alpha}^{\kappa}, S_{z}\right]=0$. The matching condition (23) is not affected, since the leading contributions at small $R$ of both the outside and the inside parts of (16) are independent of $\kappa$. We obtain the eigenfunctions for $\kappa \neq 0$ from those for $\kappa=0$ given by (24)-(29) replacing $E$ by $E^{\kappa}-\kappa \sigma$. Thus as functions of $\vec{r}$ they are unchanged, while the eigenvalues $E^{\kappa}=E+\kappa \sigma$ of $H_{\alpha}^{\kappa}$ are shifted.

The eigenfunctions with $E^{\kappa}=\kappa \sigma$ are now the supersinglets. The other eigenfunctions are still paired by $Q_{\alpha}$ and $Q_{\alpha}^{\dagger}$, although having different energy eigenvalues. One might object that, since the superoperators no longer commute with the Hamiltonian, the pairing is not enforced as for the $\kappa=0$ case, where the pairing has effectively determined the correct "boundary conditions". Then additional unpaired 
eigenfunctions could enter the spectrum for $\kappa \neq 0$. This is not true, however. Additional eigenfunctions would have to disappear abruptly in the limit $\kappa \rightarrow 0$. Since $\kappa \neq 0$ is a perturbative effect, one has to impose a continuous limit $\kappa \rightarrow 0$.

\section{The singular Aharonov-Bohm system}

The special value of $B=0$ has not been covered so far, as for $r \rightarrow \infty$ we can no longer impose the vanishing of the eigenfunctions of the Hamiltonian. By the discussion of the last section only the case of $g=2$ needs to be considered here.

Lacking a knowledge of the behavior of the eigenfunctions as $r \rightarrow \infty$, we have to start with a more fundamental criterion for selecting the correct eigenfunctions. Ultimately, the set of correct eigenfunctions of the Hamiltonian has to supply a resolution of unity in the Hilbert space.

We consider the special case $\alpha=0$, where this system reduces to the free particle in two dimensions. The corresponding eigenfunctions are plane waves, not actually constituting a normalized orthogonal set. Still these eigenfunctions supply a resolution of unity in the Hilbert space of square-integrable 2-spinors $\mathcal{H}_{0}=L_{2} \otimes \mathbb{C}^{2}$ by theorems on the Fourier transform. Obviously, the behavior of the system for $r \rightarrow \infty$ does not affect the Hilbert space. A normalizable orthogonal system spanning $\mathcal{H}_{0}=L_{2} \otimes \mathbb{C}^{2}$ is given by the eigenfunctions of the Hamiltonian of the homogeneous magnetic field.

This can be generalized to $\alpha \neq 0$. We demand that the solutions of the eigenvalue problem of $H_{\alpha}^{A B}$ at $B=0$ yield a resolution of unity in the Hilbert space $\mathcal{H}_{\alpha}$ spanned by the normalized orthogonal set of eigenfunctions (24)-(29) at $B \neq 0$. For $\alpha \neq 0$ the system looks the same as for $\alpha=0$ as $r \rightarrow \infty$. Generally, the value of $B$ by which the systems might differ as $r \rightarrow \infty$ does not affect the Hilbert space. But we have shown that the Hilbert space underlying the system does depend on the value of $\alpha$, i. e. on the behavior at the origin.

According to 4.4 any system with $B \neq 0$ and $\alpha^{\prime}$ within the same equivalence class as $\alpha$ would supply a suitable reference basis. We choose $\alpha=\alpha^{\prime}$ for convenience.

The operators

$$
\begin{gathered}
H_{\alpha}^{A B}=-\frac{1}{4}\left(\frac{1}{r} \frac{\partial}{\partial r} r \frac{\partial}{\partial r}+\frac{1}{r^{2}} \frac{\partial^{2}}{\partial \varphi^{2}}\right) \\
+\theta(r-R)\left(-\frac{i \alpha}{2 r^{2}} \frac{\partial}{\partial \varphi}+\frac{\alpha^{2}}{4 r^{2}}\right)+\theta(R-r) \frac{\alpha}{R^{2}}\left(\frac{\alpha}{4 R^{2}} r^{2}+\frac{1}{2} r^{2}-\frac{i}{2} \frac{\partial}{\partial \varphi}+S_{z}\right), \\
Q_{\alpha}^{A B}=S_{+} V_{\alpha}^{A B}=S_{+} \frac{e^{-i \varphi}}{2}\left[\frac{\partial}{\partial r}-\frac{i}{r} \frac{\partial}{\partial \varphi}+\frac{\alpha}{r}+\alpha \theta(R-r)\left(\frac{r}{R^{2}}-\frac{1}{r}\right)\right] \\
\left(Q_{\alpha}^{A B}\right)^{\dagger}=S_{-}\left(V_{\alpha}^{A B}\right)^{\dagger}=S_{-} \frac{e^{i \varphi}}{2}\left[-\frac{\partial}{\partial r}-\frac{i}{r} \frac{\partial}{\partial \varphi}+\frac{\alpha}{r}+\alpha \theta(R-r)\left(\frac{r}{R^{2}}-\frac{1}{r}\right)\right]
\end{gathered}
$$

are obtained by multiplication of (14) and (15) with $\lambda^{-2}$ and $\lambda^{-1}$, respectively, and the subsequent limit $\lambda \rightarrow \infty$. These operators are now measured in units of $2 \hbar^{2} / M$ 
and $2^{1 / 2} \hbar /(M)^{1 / 2}$, respectively. The supersymmetry algebra (2) is fulfilled by $Q_{\alpha}^{A B}$, $\left(Q_{\alpha}^{A B}\right)^{\dagger}$ and $H_{\alpha}^{A B}$.

We solve the eigenvalue problem of the Hamiltonian in the form

$$
\left\langle E, \sigma, m\left|\left(H_{\alpha}^{A B}-\frac{k^{2}}{4}\right)\right| k\right\rangle=0 .
$$

A necessary condition on the solutions to yield a resolution of unity is that they have to be within the Hilbert space; i. e., all $\langle E, \sigma, m \mid k\rangle$ must be finite and at least one for a given $|k\rangle$ must be different from zero. Hermiticity of the Hamiltonian imposes a further condition on the solutions, which requires orthogonality $\left\langle k^{\prime} \mid k\right\rangle=0$ if $k \neq k^{\prime}$. Due to $\left[H_{\alpha}^{A B}, L_{z}\right]=\left[H_{\alpha}^{A B}, S_{z}\right]=0$, we use the ansatz $\Psi_{k, \sigma, m}(r, \varphi)=\psi_{k, \sigma, m}(r) e^{i m \varphi} \zeta_{\sigma}$. Inside of the flux tube we have again to solve a Kummer equation. Outside of the flux tube we have a Bessel equation for $k>0$, which reduces to the Laplace equation for $k=0$. If we impose continuity at $r=R$, from the eigenvalue equation (44) the matching condition (23) follows again. We will not combine the two linear independent solutions for $k>0$ to yield differentiable solutions at $r=R$, such that (23) would be fulfilled even before taking the limit $R \rightarrow 0$. Instead, in order to proceed coherently with the case of the additional homogeneous field we only demand continuity. The discussion based on the leading behavior of the solutions in section 4.1 is repeated literally by using only one of the linear independent solutions for $r>R$. The result is for $k>0$

$$
\begin{gathered}
\Psi_{k, \sigma, m}(r, \varphi)=\left[\theta(r-R) J_{m+\alpha}(k r)+\theta(R-r) \frac{R^{-|m|} e^{\frac{\alpha}{2}} J_{m+\alpha}(k R)}{{ }_{1} F_{1}\left(m \theta(m)+\sigma+1 / 2-\frac{R^{2} k^{2}}{4 \alpha}, 1+|m|, \alpha\right)}\right. \\
\left.\times e^{-\alpha r^{2} / 2 R^{2}} r^{|m|}{ }_{1} F_{1}\left(m \theta(m)+\sigma+1 / 2-\frac{R^{2} k^{2}}{4 \alpha}, 1+|m|, \frac{\alpha}{R^{2}} r^{2}\right)\right] e^{i m \varphi} \zeta_{\sigma}
\end{gathered}
$$

with $m+\alpha>0$ for $\sigma=-1 / 2$ and $m<0$, and $m+\alpha>-1$ for $\sigma=+1 / 2$ and $m \geq 0$;

$$
\begin{gathered}
\Psi_{k, \sigma, m}(r, \varphi)=\left[\theta(r-R) J_{-m-\alpha}(k r)+\theta(R-r) \frac{R^{-|m|} e^{\frac{\alpha}{2}} J_{-m-\alpha}(k R)}{{ }_{1} F_{1}\left(m \theta(m)+\sigma+1 / 2-\frac{R^{2} k^{2}}{4 \alpha}, 1+|m|, \alpha\right)}\right. \\
\left.\times e^{-\alpha r^{2} / 2 R^{2}} r^{|m|}{ }_{1} F_{1}\left(m \theta(m)+\sigma+1 / 2-\frac{R^{2} k^{2}}{4 \alpha}, 1+|m|, \frac{\alpha}{R^{2}} r^{2}\right)\right] e^{i m \varphi} \zeta_{\sigma}
\end{gathered}
$$

with $m+\alpha<0$ for $\sigma=+1 / 2$ and $m>0$, and $m+\alpha<1$ for $\sigma=-1 / 2$ and $m \leq 0$.

For $k=0$ the solutions are

$$
\begin{gathered}
\Psi_{k=0, \sigma=\mp 1 / 2, m}^{s}(r, \varphi) \\
=\nu\left[\theta(r-R) r^{\mp(m+\alpha)}+\theta(R-r) R^{\mp \alpha} e^{ \pm \alpha / 2} e^{\mp \alpha r^{2} / 2 R^{2}} r^{\mp m}\right] e^{i m \varphi} \zeta_{\mp 1 / 2}
\end{gathered}
$$

for $\sigma=\mp 1 / 2, \pm m \leq 0$ and $\mp(m+\alpha)>1 / 2$. There are three types of solutions for $k=0$ concerning their normalization behavior. 
i) We have $\nu=R^{ \pm(m+\alpha)-1} \pi^{-1 / 2}\left[( \pm(m+\alpha)-1)^{-1}+( \pm \alpha)^{ \pm m-1} e^{ \pm \alpha} \gamma(\mp m+1, \pm \alpha)\right]^{-1 / 2}$ for $\mp(m+\alpha)>1$ and $\sigma=\mp 1 / 2$. The normalized eigenfunctions can be found by directly performing the limit of the vanishing homogeneous field, i. e. $\lambda \rightarrow \infty$ in the normalized eigenfunctions in (27) and (29). The modulus squared is still a delta convergent function.

ii) We have $\nu=\left[-\pi \ln R^{2}\right]^{-1 / 2}$ for $\mp(m+\alpha)=1$ and $\sigma=\mp 1 / 2$. The eigenfunctions occur for integer $\alpha \neq 0$ and they correspond to the only eigenfunction in the $(m+\sigma)$ vacancy in the case of the additional homogeneous magnetic field. However, they are not obtained by the limit $\lambda \rightarrow \infty$ from the normalized functions (26) and (28), since they yield a logarithmically divergent norm. $\nu$ is determined by demanding that $\left\langle\Psi_{E=0, \sigma, m}^{s, \lambda} \mid \Psi_{k=0, \sigma, m}^{s, \lambda^{\prime}=\infty}\right\rangle=1$ for arbitrary $\lambda \neq \infty$. Still as for finite $\lambda$, the modulus squared yields a delta convergent function, since the weak logarithmic divergent behavior as $r \rightarrow \infty$ is compensated by any decaying test function.

iii) For $1>\mp(m+\alpha)>1 / 2$ and $\sigma=\mp 1 / 2$ we put $\nu=1$ for convenience. These solutions share their property of not being normalizable with the continuum states with $k>0$.

The eigenfunctions (45)-(47) constitute a resolution of unity in $\mathcal{H}_{\alpha}$. This follows, on the one hand, from the fact that the eigenfunctions of the free particle represent a resolution of unity in $L_{2} \otimes \mathbb{C}^{2}$. In the case of integer $\alpha \neq 0$ both for finite $\lambda$ and $\lambda \rightarrow \infty$ the vacancy occurs for the same $(m+\sigma)$-value. On the other hand, the singular eigenfunctions for $\lambda \rightarrow \infty$ occur for the same quantum numbers $m$ and $\sigma$ as for finite $\lambda$. They yield $\left\langle\Psi_{\sigma^{\prime}, m^{\prime}}^{s, \lambda^{\prime}=\infty} \mid \Psi_{\sigma, m}^{s, \lambda}\right\rangle=\delta_{m, m^{\prime}} \delta_{\sigma, \sigma^{\prime}}$ for all finite $\lambda$ and, therefore, in the finite subspace of the singular wave functions we have a resolution of unity. Since the singular eigenfunctions and the non-singular ones are mutually orthogonal by (38), the corresponding resolutions of unity do not interfere and the sum of both yields a resolution of unity of the entire $\mathcal{H}_{\alpha}$.

Having only a finite number of eigenfunctions with $k=0$, we define $I^{A B}(\alpha)$ as the number of eigenfunctions for $k=0$ and $\sigma=-1 / 2$ minus those for $k=0$ and $\sigma=+1 / 2$. It is given by

$$
I^{A B}(\alpha)=\left\{\begin{array}{lll}
n & \text { for } \alpha \in[n-1 / 2, n+1 / 2) & n=-1,-2, \ldots \\
0 & \text { for } \alpha \in[-1 / 2,1 / 2] & \\
n & \text { for } \alpha \in(n-1 / 2, n+1 / 2] & n=1,2, \ldots
\end{array}\right.
$$

sketched in figure 2 on the right hand side. $I^{s}(\alpha)$ is the same as for the nonvanishing homogeneous field. (48) coincides with the Atiyah-Patodi-Singer index theorem for compact manifolds with boundary, according to [14 by use of the appropriate boundary conditions. For integer $\alpha$ both $I^{s}(\alpha)$ and $I^{A B}(\alpha)$ give the same value $\alpha$ in agreement with the Atiyah-Singer index theorem [9] valid on compact manifolds without boundaries, which is plausible since in this case a compactification is possible. 


\section{Concluding remarks}

Our results do not depend on our special arrangement of the magnetic field, i. e. regularization. Smearing out the discontinuity of the magnetic field between $R-\eta$ and $R$ adds to the matching condition (23) a correction of $O(\eta)$ that is forced to vanish by the limit $R \rightarrow 0$.

The need for a regularization is dictated by consistency. And for this reason also the consequences of the use of a regularization are to be taken seriously. Ultimately, in what way these idealized structures could be realized in nature is yet another question to be resolved by experiment.

In view of the quantum Hall effect for $\alpha=1,2, \ldots$ the eigenfunctions of Laguerre type preserve the structure of the lowest Landau level of the homogeneous field $\alpha=0$ supplying the states to be occupied for the incompressible quantum fluid. The singular states are orthogonal to these, thus not participating. If the singular states remained unoccupied, the number of electrons per non-singular flux quantum would be the same as for $\alpha=0$. However, the total magnetic flux would be increased by the contribution of the singular flux tube. In other words if there were a singular flux tube without occupation of the corresponding singular states, the filling fraction would be effectively decreased.

Finally, this system might be interesting as a toy model for supersymmetric field theories, for two reasons. First, because the singular flux parametrizes different topological situations and thereby controls the subspace of supersinglets, related to spontaneous breaking of supersymmetry. Second, the deviation from $g=2$ provides an explicit breaking of supersymmetry from effective physics such as QED corrections at lower scales $\lambda_{C}$ than the scale $\lambda$ of the quantum mechanical system.

\section{Acknowledgment:}

I am indebted to D. Schiller and H. D. Dahmen for helpful discussions. 


\section{References}

[1] L. Page, Phys. Rev. 36, 444 (1930).

[2] L. D. Landau, E. M. Lifshitz: Quantum Mechanics, Pergamon Press (1965).

[3] R. B. Laughlin, Phys. Rev. Lett. 50, 1395 (1983).

[4] R. E. Prange, S. M. Girvin (editors): The Quantum Hall Effect, Springer (1987).

[5] J. K. Jain, Phys. Rev. Lett. 63, 199 (1989).

[6] R. R. Lewis, Phys. Rev. A 28, 1228 (1983).

[7] Y. Aharonov, D. Bohm, Phys. Rev. 115, 485 (1959).

[8] A. Moroz, Phys. Rev. A 53, 669 (1996).

[9] M. F. Atiyah, I. M. Singer, Bull. Am. Math. Soc. 69, 422 (1963).

[10] M. F. Atiyah, V. K. Patodi, I. M. Singer, Math. Proc. Camb. Phil. Soc. 77, 43 (1975).

[11] Y. Aharonov, A. Casher, Phys. Rev. A 19, 2461 (1979).

[12] R. Jackiw, Phys. Rev. D 29, 2375 (1983).

[13] M. Stone, Ann. Phys. 155, 56 (1984).

[14] P. Forgacs, L. O'Raifeartaigh, A. Wipf, Nucl. Phys. B 293, 559 (1987).

[15] L. E. Gendenshtein, I. V. Krive, Soviet Physics Uspekhi 28, 645 (1985).

[16] M. Alford, F. Wilczek, Phys. Rev. Lett. 62, 1071 (1989).

[17] Ph. de Sousa Gerbert, Phys. Rev. D 40, 1346 (1989).

[18] J. von Neumann: Mathematical foundations of quantum mechanics, Princeton University Press (1955).

[19] H. Nicolai, J. Phys. A 9, 1497 (1976).

[20] E. Witten, Nucl. Phys. B 185, 513 (1981).

[21] I. M. Gel'fand, G. E. Shilov: Generalized Functions, Vol. I, Academic Press (1964).

[22] M. Abramowitz, I. A. Stegun (editors): Handbook of Mathematical Functions, Dover Publications Inc. (1965).

[23] G. Szegö: Orthogonal Polynomials, Colloquium Publications, American Mathematical Society (1978).

[24] T. Eguchi, P. B. Gilkey, A. J. Hanson, Phys. Rep. 66, 213 (1980).

[25] E. Witten, Nucl. Phys. B 202, 253 (1982).

[26] R. G. Newton, Phys. Rev. 96, 523 (1954).

[27] B. Jancovici, Phys. Rev. D 187, 2275 (1969).

[28] J. Schwinger: Particles, Sources and Fields, Vol. III, Addison-WesleyPublishing Company (1989). 


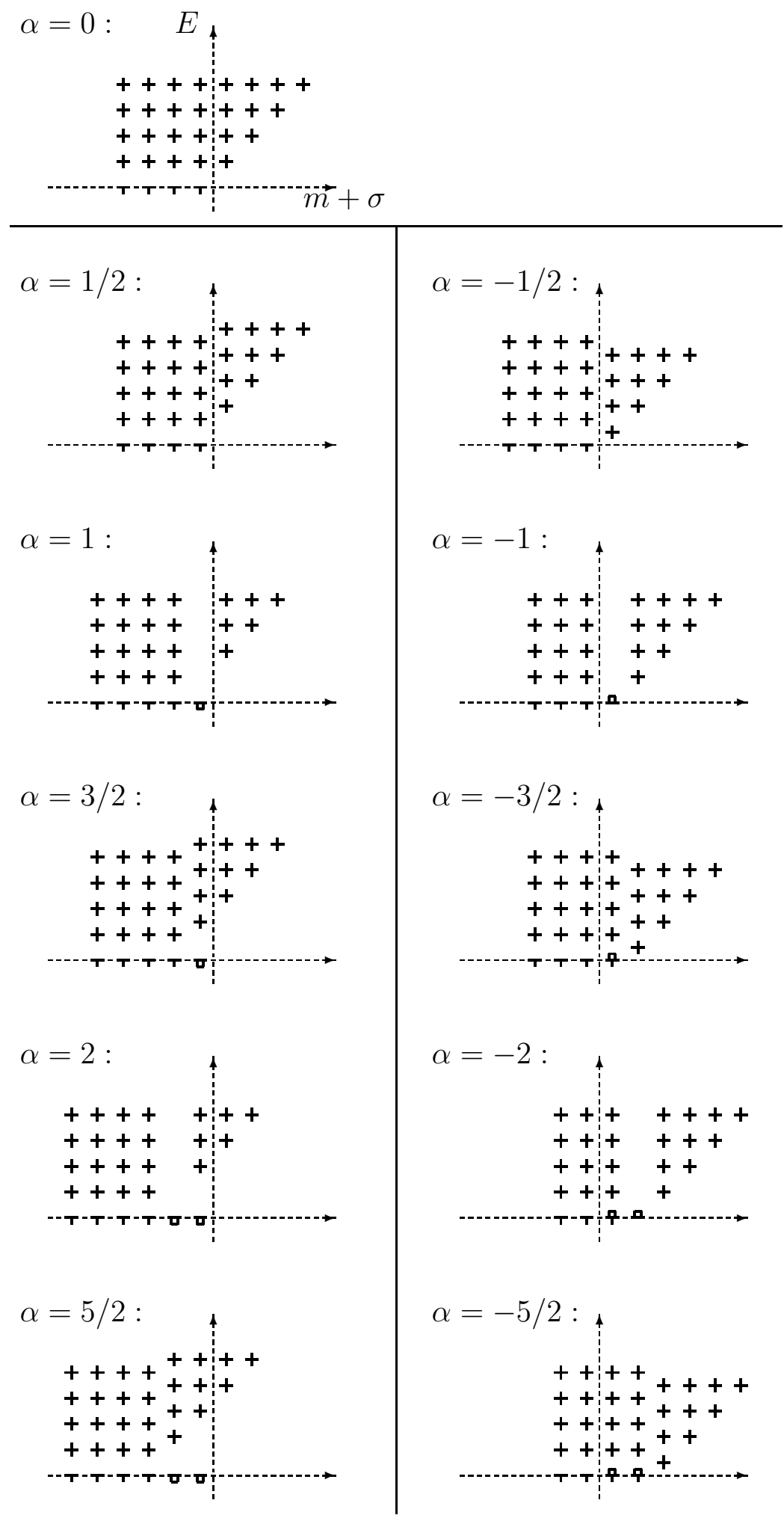

For caption see next page. 
FIG. 1 (previous page). Spectrum of $H_{\alpha}$

A state of Laguerre type with $\sigma=-1 / 2$ is indicated by $\boldsymbol{\tau}_{\mathbf{T}}$ and a state of Laguerre type with $\sigma=+1 / 2$ by $\perp$. A supersymmetric pair of such states is indicated by + . Singular states with $\sigma=-1 / 2$ are indicated by $\mathbf{z}$ and singular states with $\sigma=+1 / 2$ by $\mathbf{n}$. For $\alpha=0$ the eigenvalues of $H_{\alpha}$ constitute a regular rectangular lattice within a $3 / 8$-sector of the $E$ - $(m+\sigma)$-plane. The rectangular lattice acquires a line defect for $\alpha \neq 0$. For integer $\alpha \neq 0$ the defect is a vacancy line between the left and the right part of the spectrum, i. e. one $(m+\sigma)$-value is missing, entailing the absence of the corresponding Fourier components $\sim e^{i m \varphi}$ among the eigenfunctions of the Hamiltonian. For non-integer $\alpha$ there is a vertical shift of the right block against the left block by the non-integer part of $\alpha$. All non-integer values of $\alpha$ between two integers show the same qualitative pattern differing only in the amount of vertical shift of the right block. The spectrum is given for half-integer values. 

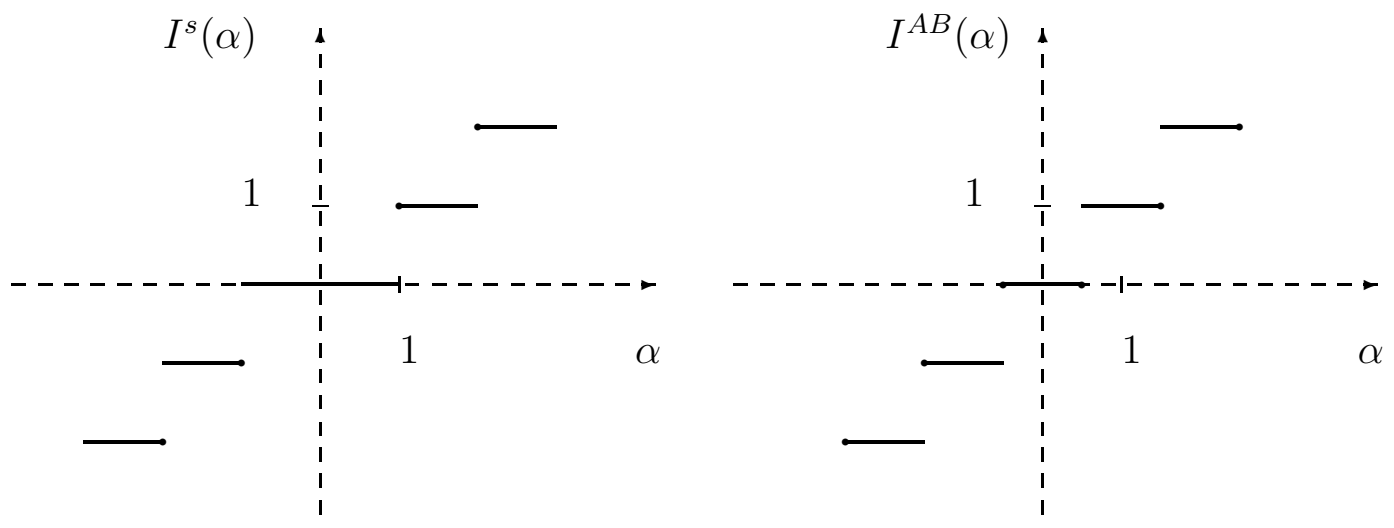

FIG. 2. The dot - indicates the value of $I(\alpha)$ at the steps. 